\title{
3D VIDEO CODING USING REDUNDANT-WAVELET MULTIHYPOTHESIS AND MOTION-COMPENSATED TEMPORAL FILTERING
}

\author{
Yonghui Wang, Suxia Cui, and James E. Fowler \\ Department of Electrical and Computer Engineering \\ Engineering Research Center \\ Mississippi State University, Mississippi State, MS
}

\begin{abstract}
A video coder is presented that combines mesh-based motion-compensated temporal filtering, phase-diversity multihypothesis motion compensation, and an embedded $3 D$ wavelet-coefficient coder. The key contribution of this work is the introduction of the phasediversity multihypothesis paradigm into motion-compensated temporal filtering, which is achieved by deploying temporal filtering in the domain of a spatially redundant wavelet transform. A regular triangle mesh is used to track motion between frames, and an affine transform between mesh triangles implements motion compensation within a lifting-based temporal transform. Experimental results reveal that the incorporation of phase-diversity multihypothesis into mesh-based motion-compensated temporal filtering significantly improves the rate-distortion performance of the $3 D$ video coder.
\end{abstract}

\section{INTRODUCTION}

It has been generally recognized that the goal of highly scalable video representation is fundamentally at odds with the traditional motion-estimation/motion-compensation (ME/MC) feedback loop which hinders the achieving of a high degree of resolution, temporal, and fidelity scalability. Consequently, the use of 3D transforms, which break the ME/MC feedback loop, are becoming the preferred approach to full scalability, and a number of modern 2D still-image algorithms have been straightforwardly extended to the third dimension (e.g., 3D-SPIHT [1]) by employing separable 3D wavelet transforms. This approach usually involves a waveletpacket subband decomposition wherein a group of frames is processed with a temporal transform followed by spatial decomposition of each frame. However, without MC, temporal transforms produce low-quality temporal subbands with significant "ghosting" artifacts [2] and decreased coding efficiency. Consequently, there has been significant interest in motion-compensated temporal filtering (MCTF) [2-9] in which it is attempted to have the temporal transform follow motion trajectories.

In this paper, we describe a $3 \mathrm{D}$ video coder using a $3 \mathrm{D}$ wavelet transform with MCTF. The salient aspect of this coder lies in that we employ multihypothesis motion compensation (MHMC) within the MCTF to combat the uncertainty inherent in estimating motion trajectories for MCTF, thereby achieving rate-distortion performance significantly superior to the usual single-hypothesis MCTF approach. Although multihypothesis has been used in conjunction with MCTF before (e.g., [8] and [9] propose both spatially and temporally diverse multihypothesis MCTF predictions), in our proposed system, we employ a new class of MHMC - phase-diversity multihypothesis [10]. Specifically, phase-diversity MHMC is implemented by deploying MCTF in the domain of a spatially redundant wavelet transform such that multiple hypothesis temporal filterings are combined implicitly in the form of an inverse transform. In essence, we combine the redundant-wavelet-multihypothesis (RWMH) paradigm we introduced in [10] with the 3D MCTF architecture emerging as the preferred approach to fully scalable video coding.

\section{REDUNDANT-WAVELET MULTIHYPOTHESIS}

MHMC [11] forms a prediction of pixel $s(x, y)$ in the current frame as a combination of multiple predictions in an effort to combat the uncertainty inherent in the ME process. Assuming that the combination of these hypothesis predictions is linear, we have that the prediction of $s(x, y)$ is

$$
\tilde{s}(x, y)=\sum_{i} w_{i}(x, y) \tilde{s}_{i}(x, y)
$$

where the multiple predictions $\tilde{s}_{i}(x, y)$ are combined according to some weights $w_{i}(x, y)$. A number of multihypothesis techniques for MC have been proposed in the past including fractional-pixelaccurate MC, B-frames, overlapped block MC, and multiple reference frames. These techniques employ multiple predictions that are diverse spatially or temporally to improve the overall predictive ability of the system. In [10], we introduced a new class of MHMC, phase-diversity MHMC, in which the multihypothesisprediction concept in extended into the transform domain. Specifically, we performed ME and MC in the domain of a redundant, or overcomplete, wavelet transform, and used multiple predictions that were diverse in transform phase.

The redundant discrete wavelet transform (RDWT) is an approximation to the continuous wavelet transform that, in essence, removes the downsampling operator from the traditional critically sampled transform to produce an overcomplete representation. As illustrated in Fig. 1, the size of each subband of an RDWT is the same as that of the input signal. Additionally, a $J$-scale RDWT can be considered to be composed of $4^{J}$ distinct critically sampled transforms, each corresponding to the choice between evenand odd-phase subsampling in both the horizontal and vertical directions at each scale of decomposition. In the RWMH paradigm outlined in [10], each one of these critically sampled transforms "views" motion from a different perspective and thus forms an independent hypothesis of the true motion of the video sequence. The inverse RDWT combines these multiple hypotheses into a single prediction. In the system of [10], this prediction is incorporated into the MC feedback loop of a hybrid video-coding architecture employing block-based ME/MC. Below, we introduce the RWMH concept into the MCTF framework to eliminate the MC feedback loop and produce a $3 \mathrm{D}$ video coder. 


\section{MOTION-COMPENSATED TEMPORAL FILTERING}

Two families of approaches to MCTF have arisen thus far. The first family of algorithms combines traditional block-based MC with temporal filtering [3-6]. These techniques have traditionally encountered a number of drawbacks in that 1) the rigid blockmotion model fails to capture all aspects of the motion field, leaving significant numbers of pixels "unconnected" between frames; 2) sub-pixel accuracy while maintaining invertibility of the temporal transform is difficult; and 3) implementation of temporal filters other than the simple Haar is hindered by the numerous unconnected pixels. The second family of MCTF schemes recognizes that a lifting implementation of the temporal transform permits MC schemes more general than block displacement to be implemented in an easily inverted fashion [7].

For example, let $x_{1}(m, n)$ and $x_{2}(m, n)$ be two consecutive frames of a video sequence, and let $W_{i, j}$ denote the operator that maps frame $i$ onto the coordinate system of frame $j$ through the particular MC scheme of choice. Ideally, we would want $W_{1,2}\left[x_{1}\right](m, n) \approx x_{2}(m, n)$. Haar-based MCTF would then be implemented via lifting as

$$
\begin{aligned}
h(m, n) & =\frac{1}{2}\left(x_{2}(m, n)-W_{1,2}\left[x_{1}\right](m, n)\right) \\
l(m, n) & =x_{1}(m, n)+W_{2,1}[h](m, n),
\end{aligned}
$$

where $l(m, n)$ and $h(m, n)$ are the lowpass and highpass frames, respectively, of the temporal transform [7]. This formulation, which can be easily extended to more complicated filters, permits any motion model to be used since the lifting decomposition is easily inverted.

In [2], it is proposed to use triangular mesh-based MC in lifting MCTF in order to circumvent the limitations of the blockdisplacement motion model. The advantages of this mesh-based approach are that the mesh provides a mapping for every pixel in the frame (i.e., no unconnected pixels) and that the motion mappings $W_{1,2}$ and $W_{2,1}$ are inverses of each other (i.e., only one field needs to be encoded). By combining this mesh-based MCTF with embedded spatial coding of the temporally transformed frames, the system of [2] has motion overhead on the order of one motion field per frame.

Below, we present a $3 \mathrm{D}$ video-coding system based upon that of [2], but with the key addition of phase-diversity multihypothesis via RWMH. As we will see in experimental results to follow, RWMH significantly improves the rate-distortion performance of MCTF.

\section{THE 3D-RWMH SYSTEM}

The encoder of our 3D-RWMH video-coding system, depicted in Fig. 2, first performs a spatial RDWT on each frame of a group of frames (GOF) and then performs MCTF in the redundant-wavelet domain. This is in contrast to many prior techniques [2-7] in which MCTF takes place in the spatial domain. Since MCTF is performed in the RDWT subbands, it is overcomplete spatially; consequently, before coding the temporal subbands, we remove this spatial redundancy by performing an inverse spatial RDWT on each frame. Finally, the GOF is coded by a suitable 3D coder (we use 3D-SPIHT [1] but other coders are possible). In essence, each RDWT phase in each frame can be considered to have viewed the MCTF from a different perspective and thus forms an independent hypothesis about the temporal filtering taking place. The inverse spatial RDWT implicitly combines these hypotheses into a multihypothesis estimate of what the true temporal filtering should be.
In our 3D-RWMH system, motion is tracked using a triangular mesh deployed in each of the subbands of the RDWT decomposition of each frame. Since all RDWT subbands are the same size, the same triangle mesh is used for all subbands of a frame. The ME procedure for the 3D-RWMH system starts by identifying a regular triangular mesh in the first frame of a GOF by dividing the frame into $16 \times 16$ blocks and splitting each block along its diagonal. Motion into subsequent frames is estimated by centering a small block at each vertex in the first frame of the GOF and finding the best matching block in the current frame. We search for the motion of the vertices by minimizing a distortion metric that spans across all subbands of the RDWT decomposition, as we did in $[12,13]$. Assuming that a GOF contains $G$ frames, this ME process results in $G-1$ motion fields, each mapping the first frame of the GOF into one of the other $G-1$ frames of the GOF, as illustrated in Fig. 3. Motion-vector information for these $G-1$ motion fields is transmitted to the decoder. The evolution of a regular triangle mesh over a GOF is illustrated in Fig. 4.

Since each of the $G-1$ motion fields are mesh-based and thus completely invertible, forward and backward motion fields between all pairs of frames in the GOF can be calculated from the $G-1$ transmitted fields. Using these forward and backward motion fields, affine transforms between the triangles of each pair of frames are used to implement a motion-compensated lifting-based filtering of the GOF in the temporal direction. This temporal filtering proceeds by mapping each triangle in a reference frame into the current frame using an affine six-parameter model as described in [14]; this affine mapping is performed for each triangle in each subband separately.

\section{EXPERIMENTAL RESULTS}

We use the "Susie" (64 frames), "Football" (96 frames), "Mother \& Daughter" (96 frames), and "Coastguard" (96 frames) sequences in our results. The first two sequences are grayscale with a spatial resolution of $352 \times 240$ pixels, and the other two sequences are grayscale with $352 \times 288$ pixels. We define each GOF to contain 16 frames. The vertices of the regular mesh are set in the first frame of each GOF on a $16 \times 16$ rectangular grid. The spatial RDWT uses the popular 9-7 biorthogonal filter, while the temporal filtering uses the 5-3 biorthogonal filter, implemented via lifting as in (2) and (3). The spatial transform uses symmetric extension at the image boundaries, while the temporal transforms uses symmetric extension at the GOF boundaries. For ME, a small block of size $17 \times 17$ is centered at each triangle vertex, and the best-match search uses integer-pixel accuracy. Since 3D-SPIHT [1], used as the core compression engine in all experiments, produces an embedded coding, each GOF of the sequence is coded at exactly the specified target rate by truncating each GOF bitstream to the prescribed rate.

We illustrate that the incorporation of phase-diversity multihypothesis into MCTF yields significant improvement in ratedistortion performance in comparison to single-hypothesis MCTF. Specifically, we compare performance of the 3D-RWMH system described above to that of a system employing more traditional MCTF filtering. That is, we compare to a system that performs MCTF in the spatial domain and then subsequently employs a critically sampled spatial transform and embedded coding. A ME procedure identical to that of our 3D-RWMH coder is employed, and, like the 3D-RWMH system, temporal decomposition takes place with 5-3 biorthogonal lifting with symmetric extension at GOF boundaries. This spatial-domain-MCTF system is essentially a single-hypothesis version of our 3D-RWMH coder and corre- 
sponds roughly to the system of [2], except that the ME process is somewhat different, and 3D-SPIHT, rather than JPEG-2000, is used to code the wavelet coefficients.

Average PSNR for the four sequences using the two algorithms is shown in Table 1. For all sequences, 3D-RWMH achieves on the order of a 1-dB gain over the system using spatial-domain MCTF. The frame-by-frame PSNR profiles in Fig. 5 also indicate that 3D-RWMH gives superior performance.

\section{CONCLUSIONS}

In this paper, we have presented a system that introduces the phasediversity multihypothesis concept into MCTF to achieve a significant improvement in rate-distortion performance in a 3D video coder. In essence, our 3D-RWMH system combines the flexibility and scalability of the lifting-based MCTF of [2] with the demonstrated performance gains associated with RWMH [10].

Currently, our 3D-RWMH exhibits significant boundary effects at GOF boundaries due to the symmetric extension employed in the temporal transform As seen in Fig. 5, the PSNR drops significantly every 16 frames, leading to annoying jittering artifacts in video playback. This well known issue can be resolved by extending the temporal filtering indefinitely in time as proposed in [15]. We are currently implementing this approach to further improve performance of our 3D-RWMH system.

\section{ACKNOWLEDGMENT}

Thanks to Z. Xiong for providing the implementation of 3D-SPIHT from [1].

\section{REFERENCES}

[1] B.-J. Kim, Z. Xiong, and W. A. Pearlman, "Low bit-rate scalable video coding with 3-D set partitioning in hierarchical trees (3-D SPIHT)," IEEE Transactions on Circuits and Systems for Video Technology, vol. 10, no. 8, pp. 1374-1387, December 2000.

[2] A. Secker and D. Taubman, "Highly scalable video compression using a lifting-based 3D wavelet transform with deformable mesh motion compensation," in Proceedings of the International Conference on Image Processing, Rochester, NY, September 2002, vol. 3, pp. 749-752.

[3] J.-R. Ohm, "Three-dimensional subband coding with motion compensation," IEEE Transactions on Image Processing, vol. 3, no. 5, pp. 559-571, September 1994.

[4] S.-J. Choi and J. W. Woods, "Motion-compensated 3-D subband coding of video," IEEE Transactions on Image Processing, vol. 8, no. 2, pp. 155-167, February 1999.

[5] B. Pesquet-Popescu and V. Bottreau, "Three-dimensional lifting schemes for motion compensated video compression," in Proceedings of the International Conference on Acoustics, Speech, and Signal Processing, Salt Lake City, UT, May 2001, vol. 3, pp. 1793-1796.

[6] P. Chen and J. W. Woods, "Bidirectional MC-EZBC with lifting implementation," IEEE Transactions on Circuits and Systems for Video Technology, August 2002, submitted.

[7] A. Secker and D. Taubman, "Motion-compensated highly scalable video compression using an adaptive 3D wavelet transform based on lifting," in Proceedings of the International Conference on Image Processing, Thessaloniki, Greece, October 2001, vol. 2, pp. 1029-1032.

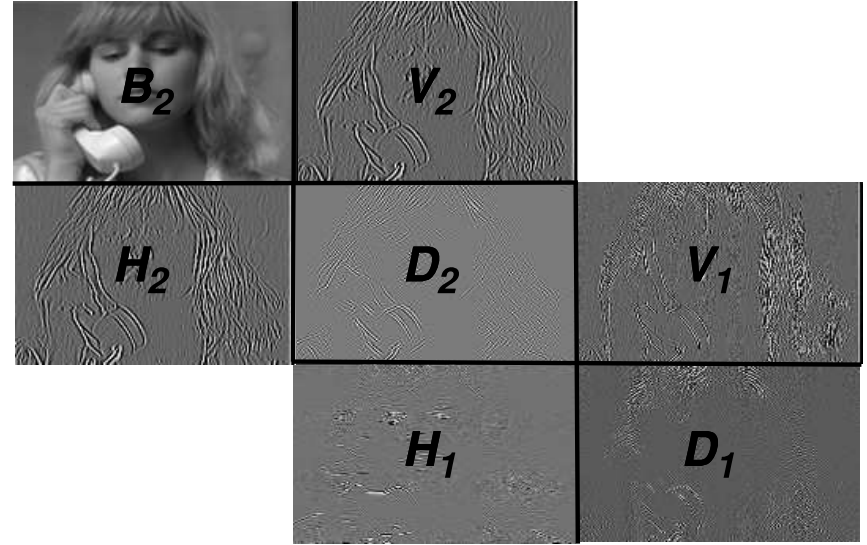

Figure 1: A two-scale RDWT of a 2D image.

[8] D. S. Turaga and M. van der Schaar, "Wavelet coding for video streaming using new unconstrained motion compensated temporal filtering," in Proceedings of the 2002 Tyrrhenian International Workshop on Digital Communications (IWDC 2002): Advanced Methods for Multimedia Signal Processing, Capri, Italy, September 2002.

[9] M. van der Schaar and D. S. Turaga, "Unconstrained motion compensated temporal filtering (UMCTF) framework for wavelet video coding," in Proceedings of the International Conference on Acoustics, Speech, and Signal Processing, Hong Kong, April 2003, vol. 3, pp. 81-84.

[10] S. Cui, Y. Wang, and J. E. Fowler, "Multihypothesis motion compensation in the redundant wavelet domain," in Proceedings of the International Conference on Image Processing, Barcelona, Spain, 2003, vol. 2, pp. 53-56.

[11] G. J. Sullivan, "Multi-hypothesis motion compensation for low bit-rate video coding," in Proceedings of the International Conference on Acoustics, Speech, and Signal Processing, Minneapolis, MN, April 1993, vol. 5, pp. 437-440.

[12] S. Cui, Y. Wang, and J. E. Fowler, "Mesh-based motion estimation and compensation in the wavelet domain using a redundant transform," in Proceedings of the International Conference on Image Processing, Rochester, NY, September 2002, vol. 1, pp. 693-696.

[13] S. Cui, Y. Wang, and J. E. Fowler, "Motion estimation and compensation in the redundant-wavelet domain using triangle meshes," IEEE Transactions on Circuits and Systems for Video Technology, October 2002, submitted.

[14] Y. Altunbasak, A. M. Tekalp, and G. Bozdagi, "Twodimensional object-based coding using a content-based mesh and affine motion parameterization," in Proceedings of the International Conference on Image Processing, Washington, DC, October 1995, vol. 2, pp. 394-397.

[15] J. Xu, Z. Xiong, S. Li, and Y.-Q. Zhang, "Memory constrained 3-D wavelet transform for video coding without boundary effects," IEEE Transactions on Circuits and Systems for Video Technology, vol. 12, no. 9, pp. 812-818, September 2002. 


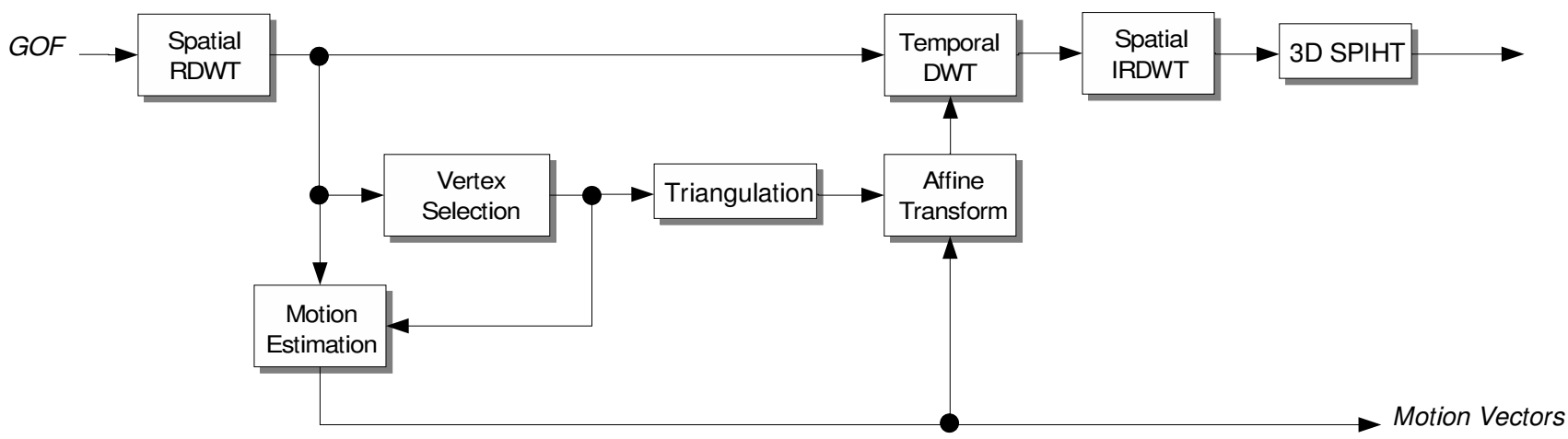

Figure 2: Block diagram of the 3D-RWMH video-coding system.
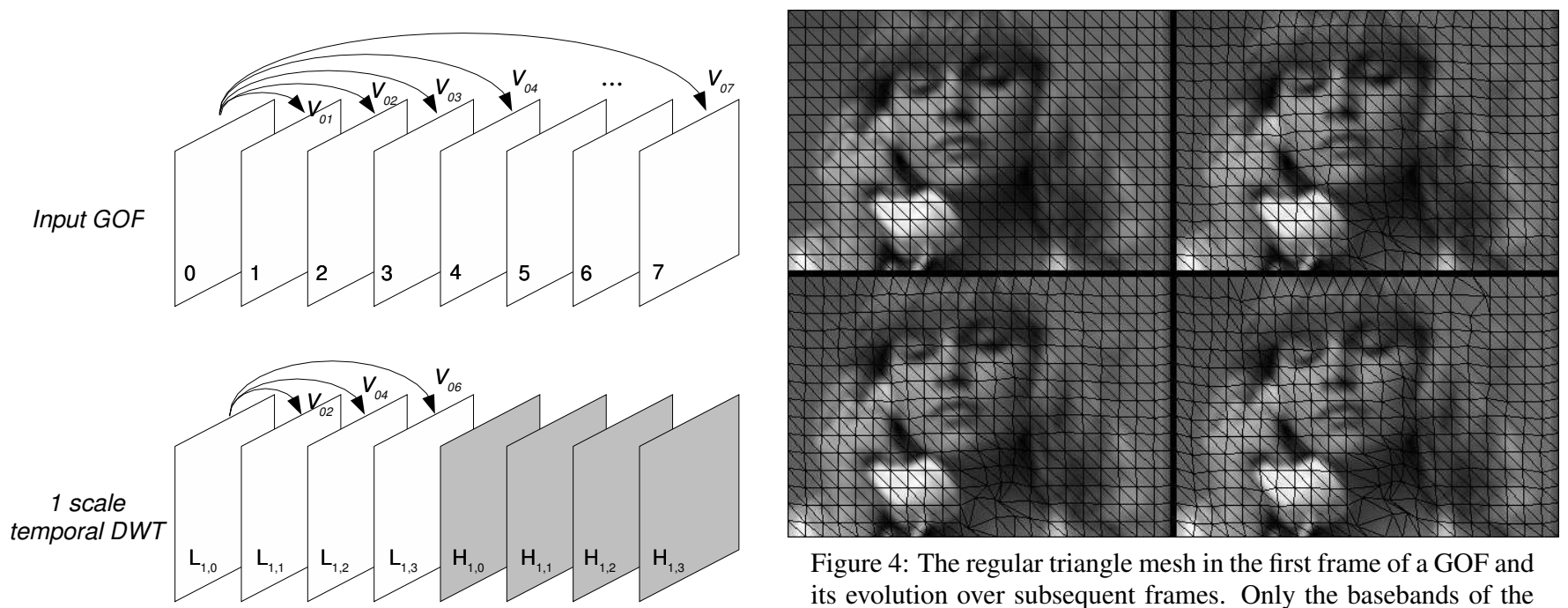

Figure 4: The regular triangle mesh in the first frame of a GOF and its evolution over subsequent frames. Only the basebands of the frames are show. Clockwise from upper-left: Frame 0, Frame 4, Frame 12, and Frame 8.

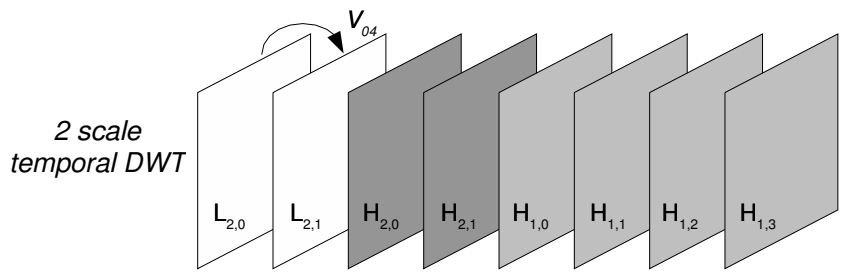

Figure 3: Motion fields for MCTF in the 3D-RWMH coder.

\begin{tabular}{|l|c|c|}
\cline { 2 - 3 } \multicolumn{1}{c|}{} & \multicolumn{2}{c|}{ PSNR $(\mathrm{dB})$} \\
\cline { 2 - 3 } \multicolumn{1}{c|}{} & $\begin{array}{c}\text { Spatial-domain } \\
\text { MCTF }\end{array}$ & 3D-RWMH \\
\hline Football $\dagger$ & 27.0 & 28.2 \\
Susie & 37.6 & 38.5 \\
Mother \& Daughter & 42.1 & 42.9 \\
Coastguard & 27.4 & 28.3 \\
\hline
\end{tabular}

Rate is 0.25 bpp except $\dagger$, which is 0.5 bpp.

Table 1: Average PSNR

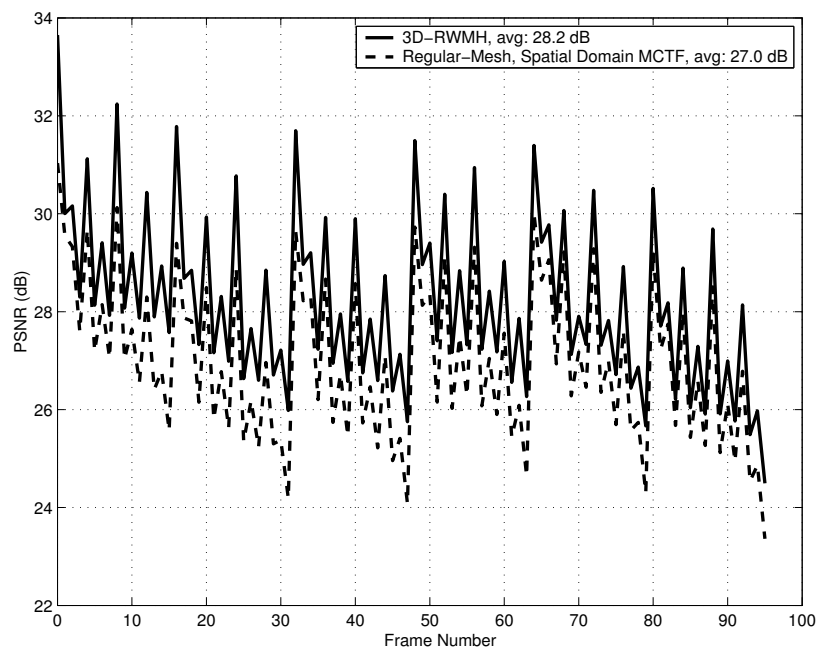

Figure 5: Frame-by-frame PSNR for "Football" at $0.5 \mathrm{bpp}$. 